\title{
Autonomous decentralized control of off-grid DC micro-grid
}

\author{
Shao-Fei Du, Yong-Qiang Zhu and Jun Wen \\ School of Electrical \& Electronic Engineering, \\ North China Electric Power University, \\ Beijing, Changping District, china \\ E-mail:dushaofei1989@163.com
}

\begin{abstract}
According to the basic idea of autonomous decentralized system, this paper divides the various units of off-grid DC micro-grid into different roles, and each roles are given specific control objectives and control strategy. In order to verify the effectiveness of the proposed off-grid DC micro-grid autonomous decentralized control strategy, this paper creates an off-grid DC micro-grid simulation model containing distributed generation, energy storage, and DC constant power load in PSCAD/EMTDC. Simulation results show that the strategy can respond quickly in the working condition of load fluctuations, energy storage reaches capacity limits, and distributed power source output fluctuation, thus enhance the stability and power supply quality of the system.
\end{abstract}

Keywords: Autonomous Decentralized Control; Off-grid DC Micro-grid; Role-Division; $\mathrm{PSCAD/EMTDC}$

\section{Introduction}

In the conventional DC micro grid control strategies, master-slave control and hierarchical control rely heavily on communication systems[1-3]. Although there are no requirements of communication, only local information, in peer-topeer control, it is not easy to achieve coordinated control between various units, and there are higher requirements on the parameters of the system, which is not conducive to practical use[4-5].

Based on the study on the basic principles of autonomous decentralized control, the paper proposes an autonomous decentralized coordinated control strategy applying to off-grid DC micro-grid.

\section{The Constitution and Operation Mode of de Micro-Grid}

\subsection{Micro-grid constitutes}

The typical structure of an off-grid DC micro-grid network is shown in Figure 1. We can classify the units into three self-node[6-8]. 


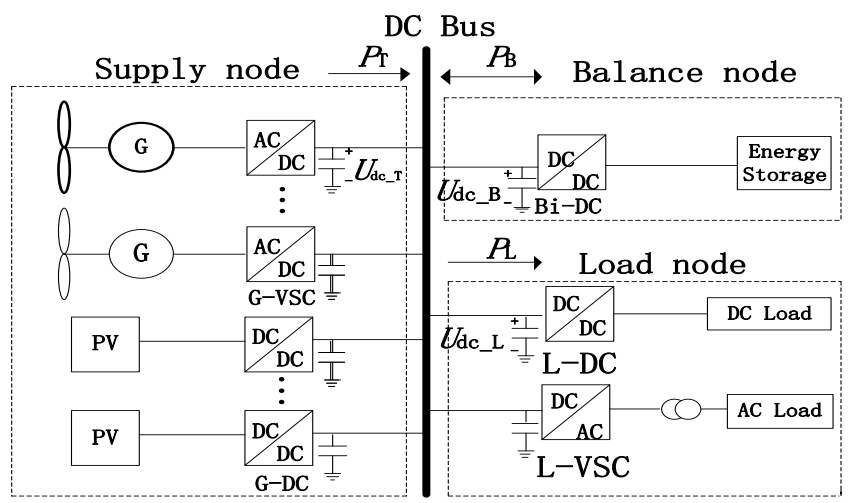

Fig. 1. Diagram of the traditional off-grid de micro-grid.

(1)Balance node

The node can absorb power form micro-grid or output power to it in order to maintain the Voltage of the grid . Its control objective is to ensure a stable voltage on the DC bus.

(2)Power supply node

The node can only output power to micro-grid. Its control objectives are to maximize the output power.

(3)Load node

The node only absorbs power from micro-grid. Its control objective is to ensure the quality and continuity of load power supply as far as possible.

\subsection{Operation mode}

Off-grid DC micro-grid ,mainly relies on the balance node to maintain the power balance of the system[9-10].

$$
P_{\mathrm{B}}=P_{\mathrm{L}}-P_{\mathrm{T}}
$$

Mode I: $P_{\mathrm{L}}>P_{\mathrm{T}}, P_{\mathrm{B}}>0$. Model II: $P_{\mathrm{L}}=P_{\mathrm{T}}, P_{\mathrm{B}}=0$. Model III $: P_{\mathrm{L}}<P_{\mathrm{T}}, P_{\mathrm{B}}<0$.

\section{Autonomous Decentralized Strategy}

Status signal and meaning shown in Table 1. 
Table 1. Status signal and meaning.

\begin{tabular}{ccl}
\hline symbol & meaning & \multicolumn{1}{c}{ Value explanation } \\
\hline & & $\mathrm{B}=1$ Energy storage device reached the storage limit \\
$\mathrm{B}$ & $\begin{array}{c}\text { Balance node state } \\
\text { information }\end{array}$ & $\begin{array}{l}\mathrm{B}=0 \text { Energy storage device proper function } \\
\mathrm{B}=-1 \text { Energy storage device reached lower limit }\end{array}$ \\
& Supply node state & $\mathrm{T}=1$ MPPT control \\
& information & $\mathrm{T}=0$ Constant voltage control \\
$\mathrm{T}$ & Load node state & $\mathrm{C}=1$ Have off-grid load \\
& information & $\mathrm{C}=0$ Haven't off-grid load \\
\hline
\end{tabular}

\subsection{Control strategy of balance node}

The Balance node ADS control flow is shown in Figure 2. DC micro-grid balance node is mainly taken on by energy storage device, and the control strategy is shown in Figure 3.

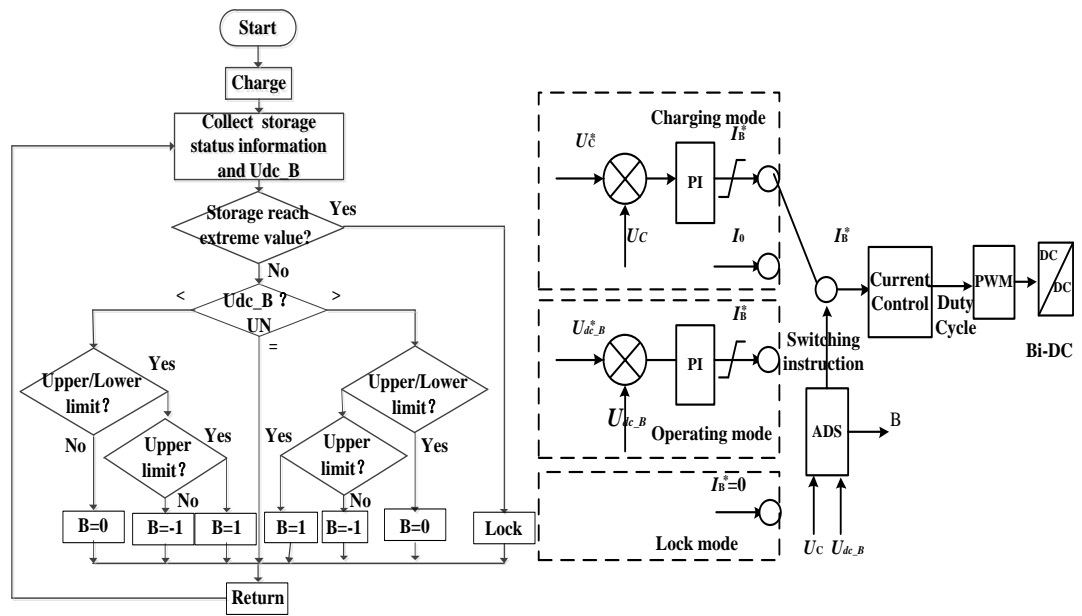

Fig. 2. ADS control flow chart of balancing bus.

Fig. 3. Control strategy diagram for Bi-DC.

\subsection{Control strategy of power supply node}

Supply node ADS control process is shown in Figure 4. Power supply nodes is undertaken mainly by various types of distributed generators. In this paper, we take solar power as an example, and the control strategy is shown in Figure 5. 

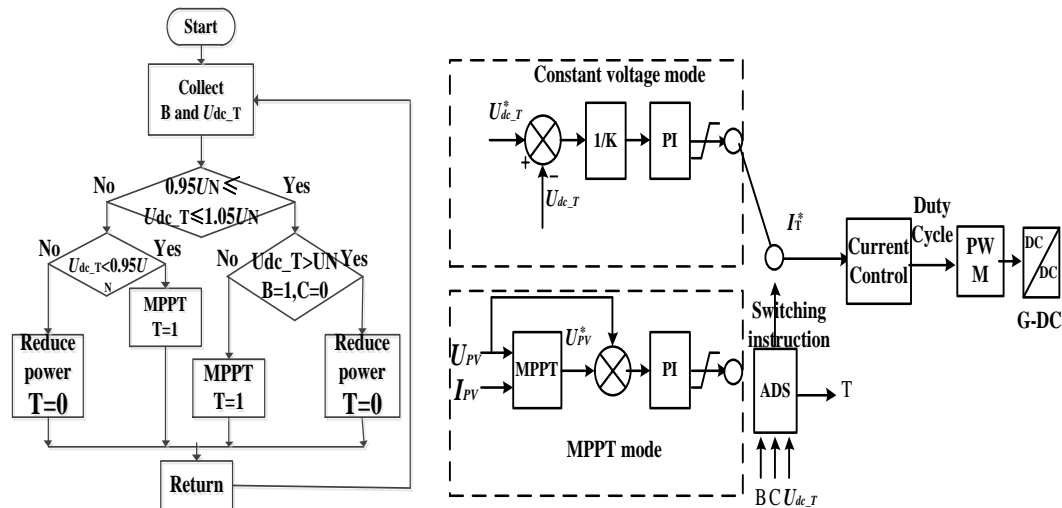

Fig. 4. ADS control flow chart of power bus Fig. 5. Control strategy diagram for G-DC

\subsection{Control strategy of load node}

Load node ADS control process is shown in Figure 6. Load nodes in DC microgrid consist of various types of AC and DC loads. This paper takes DC constant power load for example and the control strategy is shown in Figure 7.

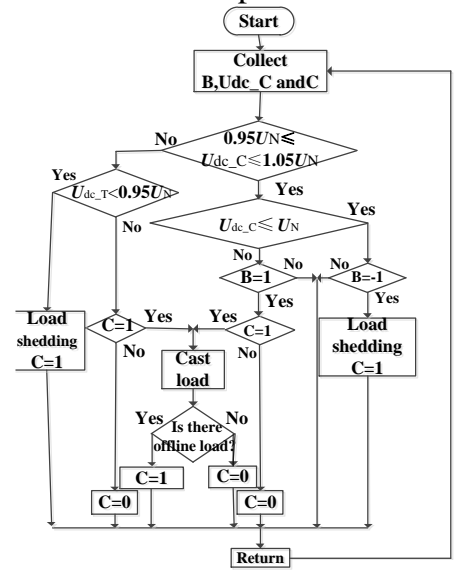

Fig. 6. ADS control flow chart of load bus

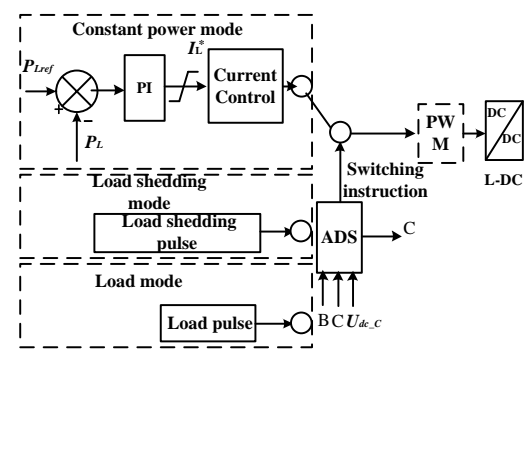

Fig. 7. Control strategy diagram for L-DC.

\section{Simulation}

In order to test and verify the effectiveness of the autonomous decentralized control strategy, we put up simulation system as shown in Figure 8 in EMTDC/PSCAD. This system includes a permanent magnet synchronous wind power generator set and a photovoltaic cell, both of whose rated power are $5 \mathrm{~kW}$. We use super capacitor as the energy storage element, capacitance value $\mathrm{C}=$ $10 \mathrm{~F}$, the upper limit of voltage $U_{\mathrm{Cmax}}=90 \mathrm{~V}$, the lower limit of voltage $U_{\mathrm{Cmin}}=50 \mathrm{~V}$, the maximum output power is given by the Eq. (2) as below. 


$$
E_{\max }=\frac{1}{2} C\left(U_{C \max }^{2}-U_{C \min }^{2}\right)
$$

We can know that when $E_{\max }=28 \mathrm{~kJ}$, loads $\mathrm{L}_{1}, \mathrm{~L}_{2}, \mathrm{~L}_{3}$ are all constant load, and the capacities are respectively $4 \mathrm{~kW}, 2 \mathrm{~kW}, 4 \mathrm{~kW}$. Among them L1 is precedence over $\mathrm{L}_{2}$ and $\mathrm{L}_{3}, \mathrm{~L}_{2}$ and $\mathrm{L}_{3}$ have the same priority. Direct-current generator rated voltage $\mathrm{UN}=400 \mathrm{~V}$. Next we give three operating modes in offgrid mode, and the simulation result of DC micro-grid with autonomous decentralized control is shown in Figure 9. We can learn from Figure 9 that in the initial state of the simulation $(0-2 \mathrm{~s})$, the power output $P_{\mathrm{T}}$ of the supply node is $10 \mathrm{~kW}$, and the system runs at the maximum power mode. When the output power $P_{\mathrm{B}}$ of the balance node is $0 \mathrm{~kW}, \mathrm{SOC}$ initial value is $70 \%$. Constant power load $\mathrm{L}_{1}, \mathrm{~L}_{2}, \mathrm{~L}_{3}$ all access to the system, the total load of the system $P_{\mathrm{L}}$ is $10 \mathrm{~kW}$, and the system voltage $U_{\mathrm{dc}}$ stable is kept at $1 \mathrm{pu}$.

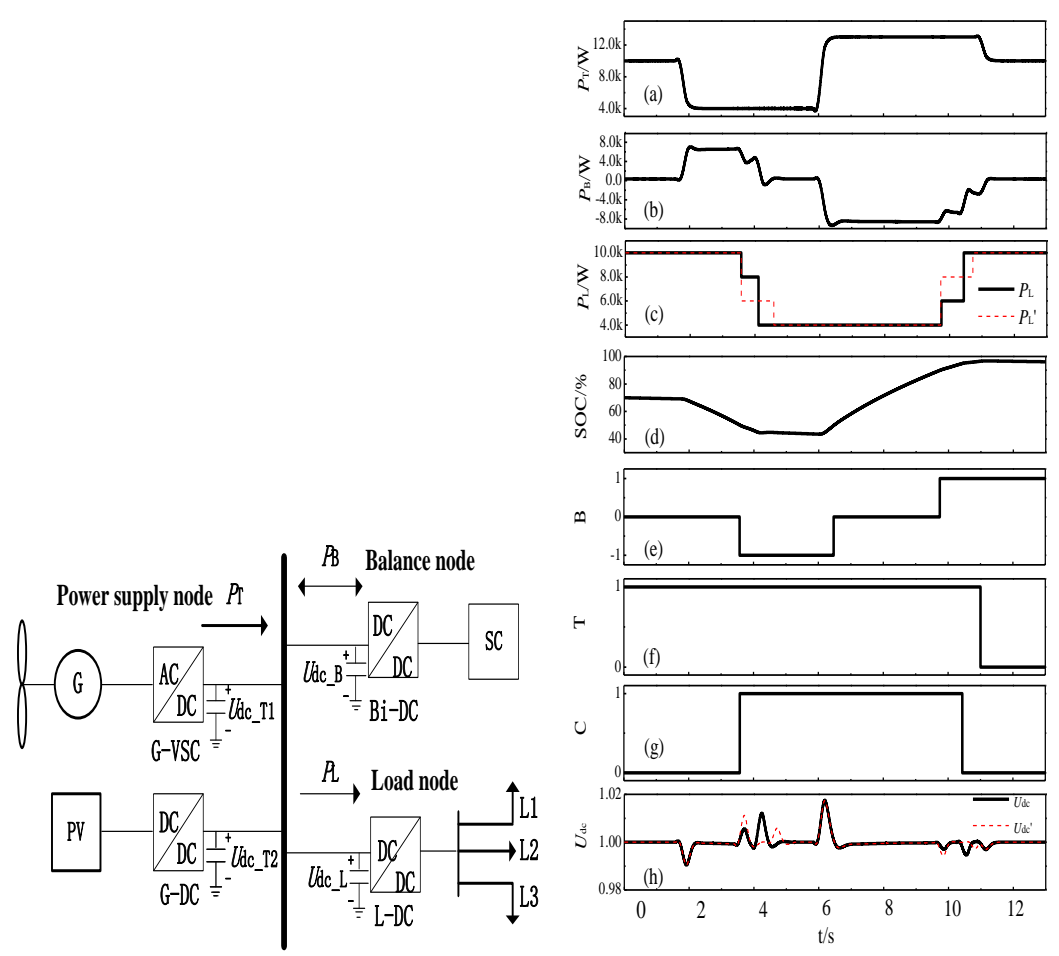

Fig. 8. Simulated system diagram of de micro-grid. Fig. 9. Off-grid dc micro-grid performances.

Mode I(2-4s): At $2 \mathrm{~s}$, power supply node output power $P_{\mathrm{T}}$ decreases to $4 \mathrm{~kW}$.

The balance node output power to compensate for the power shortage, $P_{\mathrm{B}}$ is $6 \mathrm{~kW}$, and the SOC begin to decrease, as is shown in Figure 9(a),(b),(d).At $3.5 \mathrm{~s}$, SOC of the balance node drops to $50 \%$, load $\mathrm{L}_{2}$ is removed, Since at this time $P_{\mathrm{T}}$ 
is less than $P_{\mathrm{L}}$, the balance node kept on outputting power, SOC\% drops below $50 \%$, as shown in Figure 9(c),(d). At $4 \mathrm{~s}$, load $\mathrm{L}_{3}$ is cut, only with the most important load $\mathrm{L}_{1}$ left. At this moment $P_{\mathrm{T}}$ is equal to $P_{\mathrm{L}}$; The output power $P_{\mathrm{B}}$ of the balance node is $0 \mathrm{~kW}$, as is shown in Figure 9(a)-(c), and the system enters into model II.

Model II(4-6s): In model II, $P_{\mathrm{T}}$ equals to $P_{\mathrm{L}} . P_{\mathrm{B}}$ is $0 \mathrm{~kW}$, and $\mathrm{SOC}$ remains unchanged, as is shown in Figure 9

Model III(6-11s): At $6 \mathrm{~s}, P_{\mathrm{T}}$ increases to $12 \mathrm{~kW}$. At this time $P_{\mathrm{T}}$ is more than $P_{\mathrm{L}}$. The balance node absorbs power, and $P_{\mathrm{B}}$ is $-8 \mathrm{~kW}, \mathrm{SOC} \%$ began to rise, and the system enters into mode III. At about 10s ,SOC\% rises to $90 \%$, as shown in Figure 9(a)-(d). load $\mathrm{L}_{2}$ accesses to the system, At this time $P_{\mathrm{T}}$ is still more than $P_{\mathrm{L}}$, the balance nodes continues to absorb power, and SOC continues to rise, as shown in Figure 9(a)-(d). At about $10.5 \mathrm{~s}$, load $\mathrm{L}_{3}$ accesses to system. At this time all system loads access to the system with no offline load, as shown in Figure 9(c). Since at this moment is still greater than the $P_{\mathrm{L}}, \mathrm{SOC} \%$ continues to rise. At $11 \mathrm{~s}$ the power supply node makes drop power control in order to maintain the system voltage stability, as is shown in Figure 9(a).

Figure 9 (c), (h) use the dashed line to show that, when the loads have the same priority, a large load switching simulation diagram should be selected first. Figure 9(h) shows that ,in the three off-grid modes, with the change of power supply node and the load node, balance nodes continue to adjust its output power, hence maintaining the system DC voltage between $0.98 \sim 1.02 \mathrm{pu}$, which ensures the stability of the system.

\section{Conclusion}

The advantages of this control strategy lie in:

(1)In the autonomous decentralized control strategy, each element is independent and only obtains a small amount of system status information to complete the grid-connected and off-grid control, which greatly improves the system scalability and fault tolerance.

(2)Data fields and radio communication make each node take appropriate action, we can get the system running status, predict its own operation impact on system, reduce the impact on the system by selecting the optimal mode of operation .

\section{References}

1. Lu Zongxiang, Wang Caixia, Min Yong, et al. Overview on micro grid research[J], Automation of Electric Power Systems, 2007,31(19):100107(in Chinese). 
2. Zhao Rende, Wang Yong Jun, Zhang Jia Sheng, Maximum power point tracking control of the wind energy generation system with direct-driven permanent magnet synchronous generators[J], Proceedings of the CSEE, 2009, 29(27):106-111 (in Chinese).

3. Hang Li jun, Li Bin, Huang Long, et al, A multi-resonant PR current controller for grid-connected inverters in renewable energy systems[J], Proceedings of the CSEE, 2012, 32(12):51-58(in Chinese).

4. Hu Xue feng, Wang Lu, Gong Chun Ying, et al, Harmonic analysis and suppression strategies of grid current for renewable energy grid integration system[J], Proceedings of the CSEE, 2010,30(S):167-170(in Chinese).

5. Zhang Qing hai, Peng Chu wu, Chen Yan dong, et al, A control strategy for parallel operation of multi-inverters in micro grid $[\mathrm{J}]$, Proceedings of the CSEE, 2012, 32(25):126-132(in Chinese).

6. Yang Jia, Autonomous distributed power control strategies and energy coordinate Management system of micro-grid [D], Master's thesis, Southwest Jiaotong University, (2013).

7. Liu Jiaying, Han Xiaoqing, Wang Le, Zhang Peng,Wang Jing,Operation and Control Strategy of DC Microgrid, Operation and Control Strategy of DC Microgrid[J], Proceedings of the CSEE, 014,09:2356-2362(in Chinese).

8. Wang Yi, Zhang Lirong, Li Heming, Liu Junpeng, Hierarchical Coordinated Control of Wind Turbine-based DC Microgrid[J]. Proceedings of the CSEE, 2013,04:16-24+4(in Chinese).

9. Wang Panbao, Wang Wei, Meng Nina,WuYan, Unified Control Strategy of Islanding and Grid-connected Operations for DC Microgrid[J], Proceedings of the CSEE, 2015,17:4388-4396(in Chinese).

10. Jiang Chen, Zeng Guohong, Wu Xuezhi, Yang Jie, Hierarchical Control Strategy of Bipolar-Type DC Microgrid[J], Power System Technology, 2015, 08:2217-2222(in Chinese). 